\title{
Aspectos de la regulación TIC en Colombia*
}

\author{
Douglas Velásquez Jácome** \\ Álvaro Hernán Moreno Duran ${ }^{* * * * *}$
}

Recibido: 3 de junio de 2015 - Revisado: 10 de mayo de 2015

Aprobado: 9 de septiembre de 2015

\section{Resumen}

Una de las garantías del estado social de derecho y democrático condensado en la sociedad de la información es que los beneficios de las tecnologías de la información y las comunicaciones-TIC sean accesibles a todos los ciudadanos en condiciones de igualdad.

$\mathrm{El}$ sector TIC es bastante denso por tener un gran número de subsectores en continua evolución, generando una cantidad importante de datos que corren por las redes hacia los terminales móviles de los usuarios. ¿En cuáles de ellos debería intervenir la regulación? ¿En cuáles no debe intervenir, dejando que la desregulación actúe para lograr un adecuado desarrollo de la tecnología y una mayor competencia sana que conlleve más penetración de los bienes y servicios que demande el mercado permitiendo la disminución de la brecha tecnológica entre los ciudadanos?

Palabras clave: TIC, PND, UIT, CRC, innovación, usuario, convergencia, movilidad, operadores, regulación, regulador, espectro, datos, informática, banda ancha, fibra óptica.

"Artículo producto del proyecto de investigación: "Regulación de las Nuevas Tecnologías en Colombia: Del Reloj de Dick Tracy a la Inteligencia Artificial", el cual hace parte de los proyectos de investigación institucionales de la facultad de Derecho de la Universidad Santo Tomas (Sede Bogotá). El mencionado proyecto de investigación es financiado por la Universidad Santo Tomas (Sede Bogotá).

* Doctor en Derecho y Ciencia Política. Profesor Facultad de Derecho, Universidad Santo Tomás. Correo electrónico: douglasvelasquez@usantotomas.edu.co

**- Doctor en Sociología - Universite De Paris VIII. Docente de la Universidad Santo Tomas. Correo electrónico: alvaromoreno@usantotomas.edu.co 


\title{
Aspects of ICT regulation in Colombia
}

\begin{abstract}
One of the guarantees of the social and democratic rule of law that is focused in the information society is that the benefits of the Information and Communication technology- ICTs are accessible to all citizens on an equal footing.

The ICT sector is quite dense for having a large number of subsectors continually evolving, generating a significant amount of data running through the networks to user mobile terminals. Which of them should regulation intervene? In which should not intervene, allowing the deregulation to take action for proper development of technology and more healthy competition that involves more penetration of the goods and services that the market demands enabling the reduction of the technological gap between citizens?
\end{abstract}

Keywords: ICT, PND, ITU, CRC, innovation, user, convergence, mobility operators, regulation, regulatory, spectrum, data, computer, broadband, fiber optics.

\section{Aspectos da Regulação TIC na Colômbia}

\section{Resumo}

Uma das garantias do estado social do direito e democrático condensado na sociedade da informação é que os benefícios das tecnologias da informação e as comunicações (TICs) sejam acessíveis a todos os cidadãos em condições de igualdade.

A área das TICs é bastante densa por ter um grande número de subáreas em contínua evolução, gerando uma quantidade importante de dados que percorrem as redes em direção aos terminais móveis dos usuários. Em quais deles deveria haver uma regulação? Em quais não deve intervir, deixando que a falta de regulação atue para conseguir um desenvolvimento adequado da tecnologia e uma maior concorrência que implique em maior penetração de bens e serviços na sociedade, permitindo a diminuição da brecha tecnológica entre os cidadãos e o mercado?

Palavras-chave: TICs, PND, UIT, CRC, inovação, usuário, convergência, mobilidade, operadores, regulação, regulador, espectro, dados, informática, banda larga, fibra ótica. 


\section{Introducción}

Entendemos por TIC las tecnologías, la información y las comunicaciones conectadas por Internet, lo cual tiene un efecto integrado significativo en la globalización y la sociedad de la información, lo cual se manifiesta como un fenómeno a través del cual se produce el conocimiento de la humanidad a nivel mundial de los eventos económicos, culturales, políticos, etc.

El corolario del respeto al derecho fundamental a la igualdad, como lo dicta la Constitución Política, comporta una exigencia a los poderes públicos para el trato igual a todas las personas en el acceso a los servicios públicos esenciales como lo son las TIC; atendiendo a su tipificación de servicio universal (Ferro C.M., 2001).

Las TIC se refieren a aquellas tecnologías o plataformas tecnológicas que permiten el adecuado manejo de todo tipo de información o datos de la que puede valerse la sociedad para mejorar sus condiciones de vida. Las TIC incorporan múltiples elementos y herramientas tanto de tecnologías duras (hardware, equipos, redes de telecomunicaciones, infraestructura), como blandas (software, aplicaciones, procesos) que permiten el manejo y aprovechamiento de información en múltiples formatos (datos, imágenes, videos, voz, texto).

Nuestra Corte Constitucional ha sido enfática en ratificar el derecho fundamental a la igualdad de todos los ciudadanos frente a la ley ${ }^{1}$; a su vez el artículo 365 C.P. establece que los servicios públicos son inherentes a la función social en Colombia y es deber del Estado asegurar la prestación eficiente a todos los habitantes del territorio nacional. En este sentido todos los ciudadanos tienen el derecho a acceder a las TIC, a comunicarse con cualquier otro usuario de la red de banda ancha de internet, como desarrollo a su garantía al "acceso universal". Adicionalmente, el artículo primero de la Constitución Política establece que $\mathrm{Co}^{-}$ lombia es un estado social de derecho, donde el ciudadano es el último fin. Nuestro foco es que se privilegie la protección del usuario, el respeto a su privacidad, el sano esparcimiento, los conceptos de valores humanos de ética, paz, derechos de la libre competencia y la libertad de empresa como valores constitucionales del desarrollo social y económico.

\footnotetext{
${ }^{1}$ Sentencia T-590/96
} 
De lo anterior se deduce que la regulación debe funcionar como herramienta para lograr la igualdad entre los ciudadanos ante la revolución digital y la globalización ${ }^{2}$ que traen las nuevas tecnologías de la información y las comunicaciones (TIC), reconocidas como vía indudable de desarrollo social y económico.

\section{Antecedentes de orden legal de las TIC}

El avance legal logrado en Colombia con la derogatoria de la antigua normatividad de las telecomunicaciones y la llegada afortunada de la Ley TIC $^{3}$ constituyó el reconocimiento por parte del Estado del mandato constitucional de protección de los derechos de usuarios/consumidores/clientes de las nuevas tecnologías a través de una política y regulación basadas en la promoción, el acceso, uso y apropiación de las tecnologías de la información y las comunicaciones (TIC). Ello debe ir acompañado con el despliegue y uso eficiente de la infraestructura requerida, el desarrollo de contenidos y aplicaciones, la educación y formación de talento humano. Las TIC son hoy la base legal para que con las tecnologías se consolide el desarrollo social, económico y la competitividad para una sociedad de la información y del conocimiento (Guerra M.R. y Oviedo, J., 2010). Sólo falta la voluntad política, pues no puede existir una desconexión entre la política pública, la norma regulatoria y el área tecnológica. En consecuencia, el Estado colombiano mediante la regulación debe intervenir las TIC, como un servicio público ${ }^{4}$, para garantizar su calidad, cobertura, prestación continua, ininterrumpida y eficiente, dado su carácter de interés público y social.

${ }^{2}$ Globalización. Comprende los cambios en la sociedad y la economía mundial, expresados generalmente, en un incremento sustancial del comercio en todos los campos posibles de la vida en comunidad y a nivel individual (Álvarez P., 2007).

${ }^{3}$ Para nosotros la normatividad de las telecomunicaciones evoluciona en Colombia desde el Decreto Legislativo 3418 de 1954, la Ley 555 de 2000 (PCS), la Ley 72 de 1989, El Decreto Ley 1900 de 1990, la Ley 37 de 1993 (telefonía móvil celular), la Ley 182 de 1995 (Servicio Público de Televisión y la Ley 1341 de 2009.

${ }^{4} \mathrm{E}$ artículo 365 C.P. establece que los servicios públicos son inherentes a la función social en Colombia y es deber del Estado, asegurar la prestación eficiente a todos los habitantes del territorio nacional. En este sentido todos los ciudadanos tienen el derecho a acceder a las TIC, a comunicarse con cualquier otro usuario de la red de banda ancha de internet, como desarrollo a su garantía al "acceso universal” 
En este sentido todos los ciudadanos tienen el derecho a ser usuarios de las TIC y a las redes de internet dentro del amparo al "acceso y servicio universal", en igualdad de condiciones como en su momento lo estableció la regulación para los servicios de telefonía pública básica conmutada (TPBC), con la diferencia que estos se tratan de servicios públicos domiciliarios ${ }^{6}$.

\section{Internet}

Las nuevas tecnologías se basan en los desarrollos del internet, el más grande descubrimiento de la humanidad en los últimos 200 años, denominada la red de redes. El Protocolo Internet (IP)(Belen M., 2009) ha conllevado la facilidad del uso de la tecnología, el acceso a la información y las comunicaciones por la sociedad, brindándole el camino al desarrollo social y económico. La información, datos o contenidos cursada por la red de internet es almacenada, procesada y compartida, creando con ello flujos que son útiles respecto de la interacción de los usuarios de las TIC también llamada electrónica, consistente en una gama amplia de servicios, aplicaciones y tecnologías, que utilizan diversos tipos de equipos y de programas informáticos, como medios de transmisión a través de las redes de telecomunicaciones.

Con el internet (Kumer, 2004) se impone un nuevo concepto regulatorio y de gobernanza de sus contenidos, servicios y para su infraestructura dentro del cual se considera entre otros el espectro electromagnético y radioeléctrico, la redes satelitales y de radio, las microondas, la fibra óptica, la banda ancha, la red de telefonía móvil celular y la antigua red fija hoy utilizada para transporte de datos. La infraestructura se refiere a toda clase de elementos físicos o equipos terminales que proveen la conectividad digital a los usuarios. Así aparece el concepto de ecosistema digital, adoptado por el Ministerio de $\mathrm{TIC}^{7}$, entendido como un conjunto de elementos que actúan armónicamente con todos sus componentes, en condiciones

${ }^{5}$ Servicio Universal, definido por Resolución 156 de 1999 de la CRT como "aquel que pretende llevar acceso generalizado a los hogares los servicios básicos de telecomunicaciones, iniciando con el servicio de telefonía y posteriormente integrando otros servicios a medida que los avances tecnológicos y disponibilidad de recursos lo permitan.”

${ }^{6} \mathrm{Al}$ autor como Director de la Comisión de Regulación de Telecomunicaciones (CRT) le correspondió coordinar junto con Diego Molano y Gustavo Peña la expedición de la Resolución CRT 087 de 1997 que abrió la competencia de la telefonía de larga distancia concentrada en un monopolio por más de 50 años en favor de la empresa estatal TELECOM.

${ }^{7}$ Uno de los objetivos estratégicos del MinTIC es lograr el completo desarrollo del Ecosistema Digital. 
de colaboración y competencia, que logra la administración, creación y difusión de conocimiento e información.

\section{El espectro electromagnetico. Concepto tecnico y legal}

El espectro electromagnético es primer elemento esencial de infraestructura TIC junto con los demás equipos físicos y dispositivos terminales que proveen la conectividad digital entre los usuarios de los servicios TIC. El espectro electromagnético es propiedad del Estado, administrado y regulado por los gobiernos de cada país, siguiendo la normalización de la Unión Internacional de Telecomunicaciones (UIT)

El artículo 75 de nuestra C.P. define el espectro electromagnético como un bien público, inajenable e imprescriptible sujeto a la gestión y control del Estado. Su uso es regulado por el legislador para dar condiciones de igualdad de oportunidades de acceso para todos los ciudadanos. Es un recurso limitado y funciona como plataforma natural fundamental en el desarrollo de actividades informativas y de las TIC. Por esta razón se requiere entonces de una oportuna, prudente y pertinente intervención regulatoria del Estado para que su acceso, administración, manejo de la información y datos trasmitidos a través de sus bandas electromagnéticas evite prácticas que faciliten la concentración monopólica en su utilización y proteja los derechos a la propiedad, igualdad y la libre competencia, así como que evite la violación del derecho a la privacidad y los delitos informáticos

\section{Espectro Radioeléctrico}

La UIT define como espectro radioeléctrico a "Las frecuencias del espectro electromagnético usadas para los servicios de difusión y servicios móviles, de policía, bomberos, radioastronomía, meteorología y fijos. Este no es un concepto estático, pues a medida que avanza la tecnología se aumentan (o disminuyen) rangos de frecuencia utilizados en comunicaciones, y corresponde al estado de avance tecnológico...". Para MINTIC el espectro radioeléctrico es “el conjunto de ondas electromagnéticas, cuya frecuencia se fija convencionalmente por debajo de 3000 $\mathrm{GHz}$, que se propagan por el espacio sin guía artificiar. El espectro radioeléctrico es elemento esencial de las telecomunicaciones y su organización se encuentra plasmada en el Cuadro Nacional de Atribución de Bandas de Frecuencias-CNABF, 
de acuerdo con las normas y prácticas tanto nacionales como internacionales, así como los desarrollos tecnológicos."

El espectro radioeléctrico, se divide en rangos de bandas de frecuencia:

- Banda UHF, ondas electromagnéticas que son utilizadas por compañías de telefonía fija y telefonía móvil, compañías encargadas del rastreo satelital de automóviles y establecimientos, y las emisoras radiales como tal.

- Banda VHF, también es utilizada por las compañías de telefonía móvil y terrestre y las emisoras radiales, además de los sistemas de radio de onda corta (aficionados) y los sistemas de telefonía móvil en aparatos voladores.

- Banda HF: Tiene las mismas prestaciones que la banda VHF, pero esta resulta mucho más "envolvente" que la anterior puesto que algunas de sus "emisiones residuales" (pequeños fragmentos de onda que viajan más allá del aire terrestre), pueden chocar con algunas ondas del espacio produciendo una mayor cobertura de transmisión.

En conclusión el espectro radioeléctrico es el medio por el cual se transmiten las frecuencias de ondas de radio electromagnéticas que permiten las telecomunicaciones (radio, televisión, Internet, telefonía móvil, televisión digital terrestre, etc.), y son administradas y reguladas por los gobiernos de cada país siguiendo los parámetros de la UIT.

\section{Mecanismos para asignación del espectro}

Con la expedición de la Ley 1341 de 2009 se dio origen a un debate sobre los mecanismos y facultades para asignar bandas de frecuencia dentro del espectro radioeléctrico, pues por regla general debe hacerse mediante procedimientos de selección objetiva fijada por el artículo 75 C.P., cuya excepción contenida en dicha ley podría resultar a primera vista contraria al mandamiento constitucional. Ello exigió un riguroso examen por parte de la Corte Constitucional ${ }^{9}$ para determinar la razonabilidad y proporcionalidad de la excepción y su posible violación de principios constitucionales de igualdad de oportunidades en el acceso al uso del espectro electromagnético, además de la violación de los artículos 29 y 333 del C.P. sobre el debido proceso y la libre competencia económica.

\footnotetext{
${ }^{8}$ Ministerio de Tecnologías de la Información. Decreto 4392 de 2010

${ }^{9}$ Corte Constitucional - Sentencia C-403/10
} 
La Corte Constitucional consideró que las excepciones previstas por el legislador para otorgar de manera directa el uso del espectro electromagnético se basa en la garantía de continuidad del servicio, habida cuenta del carácter de "inherentes a la finalidad del Estado" de los servicios públicos, frente a la obligación del Estado en la prestación eficaz de estos servicios. Por tal razón, en criterio de la Corte, la excepción resulta no sólo constitucionalmente válida, sino también importante y legítima, toda vez que la fórmula se establece para evitar la suspensión del servicio de telecomunicaciones donde, por motivos ajenos a la voluntad de la administración, esté en peligro la continuidad del mismo, lo que inevitablemente constituiría un perjuicio para los usuarios. Por la tanto, dijo la Corte, la aplicación de excepción a la selección objetiva por ampliación de cobertura o por interés general no vulnera per-se el derecho a la igualdad y la libre competencia por cuanto el legislador había previsto el otorgamiento de permisos de uso del espectro electromagnético de manera directa, por ampliación de cobertura e interés general, excepción que persigue un fin constitucionalmente válido.

\section{Servicios convergentes y móviles}

Los avances de la tecnología basados en el uso del espectro electromagnético y la fibra óptica como medio de transporte implican hoy la convergencia y movilidad de los servicios TIC.

Podríamos definir empíricamente la convergencia tecnológica como el medio o infraestructura física de redes por donde cursan todos los servicios y contenidos que ofrecen los operadores a los usuarios como la fibra óptica u otros medios como el espectro electromagnético, vinculados con los dispositivos terminales donde se reproducen, dentro de un mercado. Los Servicios convergentes hacen referencia a la capacidad de diferentes plataformas y redes para transportar diversos servicios en forma digital o de información los que son recibidos por un mismo dispositivo como el smartwatch, la televisión o un PC.

El concepto de servicios móviles, representa un avance de la generación anterior conocida como servicio de telefonía móvil celular (TMC), que hoy con los avances tecnológicos se prestan por los operadores a los usuarios comprendiendo adicionalmente los datos, entendidos como servicio de acceso al internet. En particular nosotros definimos los servicios móviles de TIC como un servicio público de telecomunicaciones no domiciliario, de ámbito y cubrimiento nacional, que 
proporciona en sí mismo capacidad completa de información y comunicación (TIC) entre usuarios móviles, $\mathrm{PC}$ o fijos a través de la interconexión de la red fija telefónica pública conmutada (RTPC), la Móvil Celular (TMC) o la red de internet (PI).

Los nuevos servicios móviles y convergentes, así como los nuevos desarrollos tecnológicos que se van sucediendo, requieren de una regulación convergente y una institucionalidad reguladora pública y de control convergente además de una estricta veeduría ciudadana que garantice la oportunidad y calidad en condiciones de eficiencia en el servicio. La convergencia y la movilidad con aplicaciones novedosas y gadgets $^{10}$ están transformando sectores tradicionales de la sociedad como el campo, la educación, la salud, los bancos, transporte, hospedaje, turismo, comercio etc.

Nos referiremos a algunos de estos desarrollos solo para mostrar cómo la política y la regulación de las TIC deben estar atentas y a la vanguardia de estos desarrollos para que TODOS los colombianos tengan igual oportunidad de acceso a estos. Para tal efecto reseñamos el alcance de algunas de estas tecnologías.

\section{La banda ancha en Colombia. Último kilómetro}

La banda ancha se refiere a conexiones de internet y su capacidad de velocidad de altos volúmenes de datos como el 'streaming' en alta definición y videojuegos en línea, entre otras aplicaciones ${ }^{11}$. El servicio de internet se presta para datos dentro de planes de ADSL+ ${ }^{12}$, fibra óptica a los hogares y banda ancha móvil o una combinación de estas. Pero por costos, en especial del denominado último kilómetro -la porción más importante de cualquier red de telecomunicaciones- se hace imposible incluir a una gran parte de la población marginada con servicio de internet de banda ancha a sus hogares o su dispositivo móvil, aunque existen experiencias exitosas en países con economías similares a la colombiana. Una combinación de fibra óptica en todas las cabeceras municipales, habitadas con ADSL+, más banda ancha móvil, más las redes de TMC podría generar un impulso enorme al poner al alcance de la mayoría de los ciudadanos los servicios de TIC (Stornelli N., 2015).

\footnotetext{
${ }^{10}$ Para nosotros los gadgets son dispositivos electrónicos portátiles como PDAs, móviles, Smartphone, USB, teclados, mouse, reproductores mp3, entre otros.

${ }^{11}$ Netflix es un servicio de películas en línea con más de medio millón de suscriptores en Colombia. Se calcula que el consumo de video en Colombia se multiplicará por cinco en dos años.

${ }_{12}$ Utilizando las líneas de cobre de la antigua telefonía fija
} 
Pero adicionalmente a lo anterior, Colombia por regulación de hace cinco años tiene una definición "pobre" de banda ancha. La CRC definió que existe banda ancha cuando una conexión tiene velocidades de 'bajada' (de información) de al menos $1.024 \mathrm{Kbps}$ (1 Mbps) y $512 \mathrm{Kbps}$ de 'subida'. La Organización para la Cooperación y el Desarrollo Económicos (OCDE) estableció que el 92 por ciento de las suscripciones del país tienen velocidades inferiores a los 4 megabits por segundo (Mbps). En la mayoría de los países de la OCDE, la banda ancha cuenta con velocidades reales superiores a los $10 \mathrm{Mbps}$. Se presenta un enorme rezago en el país en términos de velocidades del internet, responsabilidad de la CRC (Hernández, 2015). Afortunadamente la Ley Plan Nacional de Desarrollo 2014-2018, siguiendo recomendaciones de la OCDE dispuso que "la Comisión de Regulación de Comunicaciones (CRC) deberá establecer una senda de crecimiento para la definición regulatoria de banda ancha a largo plazo". Esta definición es requisito ineludible para hacer accesible a todos los usuarios/ciudadanos los últimos desarrollos tecnológicos, dentro de su derecho a la igualdad.

\section{Ultimos desarrollos TIC para los usuarios/ciudadanos}

Los desarrollos continuos y extraordinariamente rápidos de las tecnología de la Información y las comunicaciones (TIC) como el smartwatch, los pagos digitales, internet de la cosas, el big data y la inteligencia artificial, deben ser considerados como instrumentos de desarrollo social, económico de acceso universal. Reseñamos algunos.

\section{SMARTWATCH}

El dispositivo SMARTWATCH combina los servicios del antiguo reloj de Dick Tracy, el famoso detective de los "Comics" de los años 50s, pero hoy es más avanzado pues no solo se trata de llamadas de voz e imagen, sino que permite contar con dispositivos "todo en uno" al brindar la posibilidad de navegación en internet y acceder a servicios convergentes como el GPS, video por streaming, correo electrónico y redes sociales, cámara, música, USB etc. 


\section{Billetera digital}

La Billetera Digital ${ }^{13}$ se está convirtiendo en la opción preferida para las compras y financieras móviles. Son aplicaciones y hardware que permiten comprar en forma directa, sin necesidad de tarjeta de crédito y se está transformando en el principal medio de pago de cualquier suma a través de dispositivos móviles.

El 22 de octubre de 2015 se sancionó por el Presidente de la República la ley de Inclusión Financiera. La ley y su reglamentación es un tímido paso hacia la transformación tecnológica del sector financiero colombiano, pues solo permite la creación de entidades financieras que ofrecerán giros y transferencias con uso de nuevas tecnologías.

El poderoso sector financiero del país es temeroso a un real desarrollo de la Billetera Digital. El solo ingreso de nuevas entidades especializadas en Pagos, Ahorros y depósitos $\mathrm{PAD}$ operadas por cualquier entidad que cumpla con los requisitos legales y que obtenga licencia por parte de la Superintendencia Financiera, no implica sino el solo asomo a las nuevas tecnologías.

Colombia requiere de un regulador oportuno ante la necesidad de intervenir en la regulación de desarrollos tecnológicos como los medios de pagos digitales para todos los usuarios/ciudadanos, en todos los lugares del país.

\section{Internet de las cosas}

El Internet de las cosas, en inglés IoT - Internet of Things -, consiste en que los objetos que nos rodean incluyen ahora pequeñas redes de internet que nos conectan entre ellos con nosotros. El ejemplo son electrodomésticos, cortinas, bombillas, calefacción ya conectados a Internet que desde nuestro smartwatch podemos apagar o prender, o llevar el control del stock del refrigerador.

Otro ámbito de acción del IoT es el de las ciudades inteligentes o Smart Cities. En ellas el Internet de las Cosas se aprovecha para medir ciertos parámetros externos (tráfico, seguridad, temperatura, energía, actividad, luz, humedad, errores, etc.), de forma automática y sin la interacción del ser humano y que esos datos viajen a un centro de procesamiento para que se tomen las decisiones adecuadas en tiempo

${ }^{13}$ Cartera Digital https://www.skrill.com/es-us/negocio/productos/wallet/Skrill Digital Wallet permite a su empresa aceptar pagos seguros "solo con contraseña" en 40 monedas de más de 36 millones de personas de todo el mundo. 
real. Un ejemplo, son muchas las ciudades que están implementando redes de sensores en multitud de puntos como alarmas, semáforos, alcantarillas, vehículos, alumbrado para cuantificar peatones, tráfico vehicular, transporte público que pasan por un determinado cruce para optimizar automáticamente el tráfico en esa zona.

\section{Inteligencia artíficial}

La inteligencia artificial es una rama de la computación que relaciona un fenómeno natural, datos y hechos reales con una analogía artificial a través de programas de computador. La inteligencia artificial, por lo tanto, puede diseñar procesos que al ejecutarse sobre una arquitectura tecnológica producen resultados que maximizan en una cierta medida el rendimiento de sus resultados pues se basa en desarrollar sistemas expertos que pueden imitar la capacidad mental del hombre y relacionan reglas de sintaxis del lenguaje hablado y escrito sobre la base de la experiencia, para luego hacer juicios acerca de un problema, cuya solución se logra con mejores juicios y más rápidamente que el ser humano ${ }^{14}$.

En la actualidad la forma de inteligencia artificial más popular existe en los videojuegos, dado su consumo masivo. En este contexto, se aplica a enemigos, equipos de futbol, contraparte en ajedrez y personajes controlados por el computador o smartphone para que su actuación a lo largo de la experiencia interactiva resulte creíble y parezca espontánea. El tipo de inteligencia artificial utilizada en cada caso es diferente y responde a una serie de necesidades particulares.

Sin lugar a dudas, una de las aplicaciones más interesantes y significativas de la inteligencia artificial es la investigación científica. Con la ayuda de un computador o un conjunto de ellos, se potencian considerablemente las posibilidades del ser humano de descubrir los misterios que tan apasionadamente viene persiguiendo desde hace siglos.

Cuando se otorga a estos dispositivos la habilidad de aprender y de discernir, se los convierte en entidades que rozan las capacidades de un superhombre, dado que alcanzan velocidades de procesamiento imposibles para nosotros y que no necesitan descansar para funcionar, entre otras ventajas que los ubican por sobre los seres vivos en este contexto. Lo mejor es que son habilidades rápidas e incorruptibles.

\footnotetext{
${ }^{14}$ En la medicina tiene gran utilidad al acertar el $85 \%$ de los casos de diagnóstico.
} 


\section{$B I G D A T A$}

El Big Data es la gestión y análisis de enormes volúmenes de datos que superan los límites y capacidades de las herramientas de software habitualmente utilizadas para la captura, gestión y procesamiento de datos. El objetivo de Big Data, al igual que los sistemas analíticos convencionales, es convertir el Dato en información que facilita la toma de decisiones, incluso en tiempo real. Se refiere a información obtenida en diferentes redes sociales, en el número cada vez mayor de dispositivos electrónicos conectados, la explotación de sensores que permiten conocer los movimientos y hábitos de vida, de información externa de diversas fuentes, etc.

Dicho concepto engloba infraestructuras, tecnologías y servicios que han sido creados para dar solución al procesamiento de enormes conjuntos de datos como mensajes en redes sociales, señales de móvil, archivos de audio y video sensores, imágenes digitales, datos de formularios, emails, datos de encuestas, logs etc. Las empresas ya están utilizando Big Data para entender el perfil de sus clientes y correlacionados, sus necesidades y el sentir respecto a los productos y/o servicios. Esto adquiere especial relevancia ya que permite adecuar la forma en la que interactúa la empresa con sus clientes y en cómo les prestan servicio.

\section{Algunos antecedentes de la regulación de TIC}

Se considera que las regulaciones son las reglas o normas emitidas por el $\boldsymbol{E} \boldsymbol{s}$ tado para garantizar beneficios sociales; lo que permite afirmar que la regulación económica contiene las disposiciones mediante las cuales el Estado, a través exclusivamente del Presidente de la República, interviene en los mercados para fijar precios o cantidades de la producción, o establecer especificaciones técnicas, y en general restricciones que deben cumplir los ciudadanos y las empresas para participar en un mercado. Generalmente, este tipo de regulaciones se establecen en mercados relativamente concentrados. El regulador siempre debe garantizar su neutralidad para el equilibrio y la simetría del mercado, debe ser absolutamente neutral, entendido las posesiones e intereses, tratando de desarrollar su papel como un buen componedor, pero evitando a toda costa que este punto de neutralidad y claridad vaya a ser manchado por causa alguna (Velázquez, J, D. y Molano D., 1997).

Es entonces el Estado, por medio de la función de intervención del Presidente de la República, el único regulador. La jurisprudencia ha dividido la intervención 
del Estado en la economía por vía directa, cuando el Estado adopta medidas que orientan a los agentes económicos privados, o intervención indirecta por vía de gestión, o cuando el Estado se hace cargo el mismo de la intervención en las actividades económicas por medio de personas jurídicas generalmente públicas denominadas comisiones de regulación. ${ }^{15}$

Para entender el paso de las tecnologías y de la necesidad de implementar una regulación en TIC que vaya al compás de estos cambios e innovaciones tecnológicas, el Informe de los Antecedentes de la Conferencia Mundial de Telecomunicaciones Internacionales de 2012 (CMTI), Dubái de Diciembre de 2012 $2^{16}$, refiere que cuando se acordó el Reglamento de las Telecomunicaciones Internacionales (RTI) en 1988, todavía existía una clara distinción entre los diferentes servicios (teléfono, vídeo, etc.), así como entre los medios para prestarlos, tecnología que sólo permitía recibir llamadas de voz o telefax por la línea telefónica. Hoy con la innovación tecnológica, las telecomunicaciones pasaron de las redes de telecomunicaciones basadas en circuitos de par de cables de cobre ${ }^{17}$ a las redes de fibra óptica y banda ancha que transportan paquetes de datos o información, protocolo Internet (IP), de diversos contenidos y para ello utiliza la infraestructura global de fibra óptica y el espectro electromagnético, atrás explicada. Estamos en el desarrollo de las llamadas redes de la próxima generación o NGN, propicias en gran medida para la convergencia técnica. Es decir que hemos evolucionado de estructura "vertical" de las redes independientes para cada servicio a una estructura "horizontal" basada en el IP, que puede entregar muchos tipos de contenido a través de una misma plataforma, fenómeno conocido como convergencia tecnológica.

\section{Convergencia tecnológica}

En su momento las computadoras disponían en su mayoría de sus propias redes, y la radiodifusión era otro mundo separado. Hoy la convergencia de las tecnologías y redes existentes y la aparición de otras nuevas han cambiado radicalmente el mundo, cambio en que insistimos, la regulación y el regulador debe hacer participante a todos los usuarios/ciudadanos.

\footnotetext{
15 Sentencia C-150/03

16 Wcit-12 Actas Finales - Itu

${ }^{17}$ Red Telefonía Pública Básica Conmutada
} 
Esto plantea retos fundamentales para los organismos reguladores. En el caso de Colombia es necesario se acepte que la convergencia significa dejar atrás las antiguas distinciones entre servicios, y entender que en la actualidad estamos en el entorno de un nuevo tipo de servicios que origina un nuevo tipo de regulación, como lo formuló la UIT en la presentación de propuestas concretas relativas para la revisión de la normalización de la RTI durante la Conferencia Mundial de Telecomunicaciones Internacionales de 2012. ${ }^{18}$

\section{Neutralidad tecnológica}

Afortunadamente en Colombia después de muchas dificultades y bajo la tenacidad de la Ministra María Rosario Guerra, acompañada por varios líderes del sector de las telecomunicaciones se logró la expedición de la Ley 1341 de 2009, también llamada Ley $\mathrm{TIC}^{19}$. La ley fijó un nuevo marco legal y estableció un hito en la política pública sectorial del desarrollo tecnológico de movilidad y convergencia, favoreciendo a todas luces a los usuarios, inversionistas y operadores. Es el caso de la norma que se refiere a la neutralidad tecnológica, donde se dispone la garantía del Estado a la libre adopción de tecnologías que fomenten la eficiente prestación de servicios, contenidos y aplicaciones que usen TIC y garantía de la libre y leal competencia. ${ }^{20}$ De ahí parte la necesidad de respetar la "neutralidad tecnológica", esto es, tratar por igual a las diferentes tecnologías que ofrecen servicios similares u otros. Los organismos reguladores ya no pueden limitar su trabajo a los servicios de telecomunicaciones definidos de manera tradicional, sino que han de entender el amplio ecosistema de las TIC. Simplemente el regulador debe estar atento al día con los cambios tecnológicos y avanzar rápidamente en el análisis de la necesidad de propuestas regulatorias que faciliten la introducción de cambios de fondo a fin de dar cabida a la convergencia.

Se justifica que el regulador colombiano tenga una perspectiva más amplia y un enfoque centrado en las redes y de los mercados TIC, asumiendo un enfoque

${ }^{18}$ La UIT convocó la Conferencia Mundial de Telecomunicaciones Internacionales (CMTI) en Dubái (Emiratos Árabes Unidos), del 3 al 14 de diciembre de 2012. Conferencia Mundial de Telecomunicaciones Internacionales.

19 Para detalles de la historia e interpretación de los hitos de la Ley Tic ver Guerra M..R. y Oviedo, A, Juan. (2010). La ley de tecnologías de la información y las comunicaciones. Primeras edición. Colombia-Bogotá D.C.

${ }^{20}$ Ley 1341 de 2009 Artículo 2º. Nral. 6. 
que abarque los servicios, los contenidos, los derechos y responsabilidades de los operadores y de los consumidores, siguiendo las discusiones y propuestas de la UIT. ${ }^{21}$

\section{TIC servicio público}

Con la sola disposición sobre la neutralidad tecnológica de Ley TIC se dio un paso gigante al borrar el concepto de servicios públicos domiciliarios aplicable a la telefonía pública básica conmutada (TPBC) regulado en el Estatuto Telecomunicaciones contenido en el Decreto Ley 1900 de 1990 y la Ley 142 de 1994.

Este cambio trascendental indica también la modernización de los fundamentos de la política pública en $\mathrm{TIC}^{22}$ bajo el planteamiento de que son un servicio público $^{23}$, mas no de carácter domiciliario, fundamentado en que la evolución sectorial de los últimos años así como las perspectivas futuras se centran en los servicios móviles e inalámbricos en convergencia, como atrás se planteó. En este contexto, seguir concibiendo que las TIC son un servicio domiciliario, significaría una contradicción con la realidad de los desarrollos de la tecnología donde las normas legales y la regulación deben ir acordes con estos desarrollos dando vía libre a la introducción permanente de innovaciones tecnológicas y de nuevos mercados convergentes dentro de un ambiente de competencia.

\section{Conclusiones}

La evolución tecnológica tiene profundas implicaciones para los usuarios, el mercado, los organismos reguladores y, en último término, para lograr que las comunicaciones estén al alcance de todas las personas en el mundo.

El enorme incremento del número de abonados a los servicios TIC y la extensión global de la banda ancha móviles están imponiendo reformas urgentes del regulador y en la regulación, considerando que los consumidores de todo el mundo han dejado de usar sus dispositivos móviles como simples teléfonos para hablar con

${ }^{21}$ Desde 2012 se viene trabajando en un cambio fundamental del Reglamento Internacional de Telecomunicaciones (RTI), esto es, de tratar por igual a las diferentes tecnologías que ofrecen dentro de la aceptación del concepto de convergencia y Neutralidad Tecnológica.

22 Aún llamadas obsesivamente por algunos como "telecomunicaciones”.

${ }^{23}$ C.P. artículo 365 donde declara los servicios públicos como inherentes a la finalidad social del Estado. 
otras personas. Cada vez más, se espera de cualquier proveedor de servicios que sea capaz de ofrecer cualquier servicio de comunicación a cualquier ciudadano, en cualquier lugar, utilizando cualquier tipo de tecnología disponible todo a través del terminal Smartphone del usuario.

El regulador y la regulación deben trabajar porque los precios de los servicios estén dentro de un régimen tarifario proporcional al beneficio y calidad recibida y que correspondan a costos y una razonable utilidad, con la total garantía constitucional de competencia.

Ahora con la Ley TIC falta que el regulador y la regulación trabajen por el mejoramiento de la inclusión social de las TIC.

La regulación debe existir para hacer que a los servicios TIC, su calidad y costo puedan acceder aquellos ciudadanos más pobres y que habitan en lugares más alejados del país, todo en desarrollo del derecho fundamental a la igualdad y el servicio universal.

Corresponde al Estado regulador colombiano reconocer y establecer que el acceso y uso de las tecnologías de la información y las comunicaciones, el despliegue y uso eficiente de la infraestructura, el desarrollo de contenidos y aplicaciones, la protección a los usuarios, la formación de talento humano en estas tecnologías y su carácter transversal para todos los sectores de la economía, sean pilares para la consolidación de las sociedades de la información y del conocimiento, marchando hacia la inteligencia artificial.

Existe consenso sobre el hecho de que la adopción de marcos legales y de política que facilitan la convergencia entre las telecomunicaciones, los medios de telecomunicación y los servicios informáticos mejoran contundentemente el impacto de las tecnologías de la información y las comunicaciones en el desarrollo socioeconómico.

\section{Referencias}

Álvarez, P. (2007). Informes TIC y Globalización. Recuperado de http://ticsyglobalizacion. blogspot.com.co/2007/11/informe-tics-y-globalizacion.html

Cartera Digital. (s.f). Digital Wallet permite a su empresa aceptar pagos seguros «solo con contraseña» en 40 monedas de más de 36 millones de personas de todo el mundo. Recuperado de https://www.skrill.com/es-us/negocio/productos/wallet/Skrill 
Conferencia Mundial de Telecomunicaciones Internacionales. (2012). Conferencia Mundial de Telecomunicaciones Internacionales. Recuperado de http://www.itu.int/es/wcit-12/ Pages/default.aspx

Congreso de la Republica de Colombia (2015). Ley 1753 del 9 de junio de 2015. Plan Nacional de Desarrollo. Artículo 40․ Recuperado de http://www.consultorsalud.com/ sites/consultorsalud/files/ley_1753_de_2015_-_ley_del_plan_nacional_de_desarro1lo_2014_-_2018.pdf

Corte Constitucional - Sentencia C-150/03.

Corte Constitucional -Sentencia C-403 de 2012.

Corte Constitucional -Sentencia C-403/10.

Corte Constitucional -Sentencia T-590/96.

Ferro, C.M. (2001). Servicio universal en telecomunicaciones: Concepto y alcance en Argentina. Recuperado en http://www.uade.edu.ar/DocsDownload/Publicaciones/4_226_1578_STD027_2001.pdf1. Texto de Discusión N 27. ISBN 987-519-087

Guerra, M.R. y Oviedo, A. J. (2010). Primeras edición. Colombia-Bogotá.

Guerra, M.R. y Oviedo, A.J. (2010). La ley de tecnologías de la información y las comunicaciones (p. 76.). Colombia-Bogotá: Primeras edición.

Hernández, M. (26 de febrero de 2015). ¿Cuál debe ser la banda ancha mínima en Colombia? El Tiempo. Recuperado de http://www.eltiempo.com/tecnosfera/novedades-tecnologia/ cual-es-la-velocidad-de-la-banda-ancha-en-colombia-/15308078

ITU. Cumbre Mundial sobre la Sociedad de la Información. (s.f) ¿Qué es la Sociedad de la Información? Recuperado de https://www.itu.int/wsis/basic/faqs.asp?lang=es

Kumer, M. (2004). La Gobernanza de Internet. Recuperado de http://www.itu.int/itunews/ manager $/$ display.asp?lang=es\&year $=2004 \&$ issue $=06 \&$ \&ipage $=$ governance\&ext $=\mathrm{html}$

Ministerio de Tecnologías de la Información y las Comunicaciones. (s.f). Ecosistema Digital. Recuperado de http://www.mintic.gov.co/portal/604/w3-propertyvalue-634.html

Ministerio de Tecnologías de la Información y las Comunicaciones, (2010). Decreto No. 4392 de 2010. Recuperado de http://www.mintic.gov.co/portal/604/articles-3615_documento.pdf

Palacio, M.H. (1999). El Derecho de los Servicios Públicos, Biblioteca Vigente (p. 3, 6, $43,58)$.

Stornelli, N. (26 de junio de 2015). Cinco años de atraso en banda ancha. El tiempo. Recuperado de: http://www.eltiempo.com/archivo/documento-2013/DR-918262

Unión Internacional de Telecomunicaciones (s.f). Espectro Radioeléctrico. En Ministerio de Tecnologías de la Información y las Comunicaciones. Recuperado de http://www. mintic.gov.co/portal/604/w3-article-2350.html 
Velásquez, D. y Molano, D. (1997). El reto de la apertura. Primera Edición p. 455

Velásquez, D., Cortez P.G., Gutiérrez O.G. y Calle J. (2015). Facultad de Derecho, Universidad Santo Tomas, Investigación Asistida II Reglamentación de las TIC y Gobernanza de Internet. Bogotá. Colombia. 